\title{
Considering passenger choices and overtaking in the bus bunching problem
}

$\operatorname{AUTHOR}(S)$ :

Sun, Wenzhe; Schmöcker, Jan-Dirk

CITATION:

Sun, Wenzhe ...[et al]. Considering passenger choices and overtaking in the bus bunching problem. Transportmetrica B: Transport Dynamics 2018, 6(2): 151-168

\section{ISSUE DATE:}

2018

URL:

http://hdl.handle.net/2433/252361

\section{RIGHT:}

This is an Accepted Manuscript of an article published by Taylor \& Francis in Transportmetrica B: Transport Dynamics on 16 October 2017, available online: http://www.tandfonline.com/10.1080/21680566.2017.1387876; ; Tis is not the published version. Please cite only the published version.; この論文は出版社版でありません。引用の際には出版社版 をご確認じ利用ください。 
Unformatted version of: Sun, W. and Schmöcker, J-D. (2018). Considering passenger choices and overtaking in the bus bunching problem. Transportmetrica B, 6(2), 151-168.

Available from: https://doi.org/10.1080/21680566.2017.1387876

\title{
Considering Passenger Choices and Overtaking in the Bus Bunching Problem
}

\author{
Wenzhe SUN ${ }^{1}$, Jan-Dirk SCHMÖCKER ${ }^{1}$ \\ ${ }^{1}$ Department of Urban Management \\ Graduate School of Engineering, Kyoto University, Japan \\ Katsura Campus, Nishikyo-Ku, Kyoto, 615-8236, Japan \\ Email: \{wz.sun, schmoecker\}@trans.kuciv.kyoto-u.ac.jp
}

\begin{abstract}
Bus bunching is a well-known phenomenon on many bus routes where an initial delay to one service can disturb the whole schedule due to resulting differences in dwell times of subsequent buses at stops. This paper deals with the passenger behavior when there is more than one bus serving the stop, focusing on their choices and possible switching actions from the queue of the bus they are waiting to board. A parameter $\gamma$ is introduced to denote the percentage of passengers boarding the front bus of two buses boarding at the same time. Cases when overtaking is allowed or not are distinguished as this will also influence the passenger behavior. A set of discrete state equations is then implemented to obtain the departure times of the buses following the occurrence of an exogenous delay to one of the buses. Evaluation indices are introduced to measure the performance of the bus service along a corridor under different $\gamma$ levels. We find that it is advantageous to keep the percentage of passengers boarding the front bus low. Beside, overtaking is a favourable counter-measure against comparatively high frontbus preference.
\end{abstract}

Keywords: Bus Bunching, Passenger Behavior at Stops, Queue Switching, Service Regularity 


\section{Introduction}

Good public transport services are an essential part of a sustainable urban transport system, and improving public transport service quality is a major challenge for the operators and government agencies. Compared with railway and metro services, bus systems are obviously more vulnerable to reliability problems since they are exposed to urban road networks, sharing the limited road resources with other surface transport modes and suffering from traffic congestion, bad weather condition and unexpected events such as accidents. The resulting lack of service reliability is a major problem for bus passengers and service operators. A key feature of an unreliable service are the irregular arrivals of buses at stops. Irregular arrivals are undesirable for passengers because they lead to increased waiting times at initial stops as well as possibly missed connections at transfer stops so that passengers might lose their confidence in the public transport system. Studies have shown that passengers dislike their time waiting at bus stops more than they do their on-board travel time. For example Hollander and Liu (2008) found that the value of service reliability to bus passengers is four times higher than that of mean travel time. Therefore on-time/punctuality performance and headway evenness are important measures of service quality (e.g. Chen et al, 2009).

A consequence of irregular arrivals and a typical case of uneven headways is bus bunching which can be defined as the effect of two successive services of a single line arriving at stops with shorter headways than the designed one. Bus bunching may be caused by the first service being delayed due to unforeseen traffic congestion en-route or unplanned high demand at previous stops. The subsequent service then has fewer passengers to pick up at that stop and departs earlier than scheduled. At downstream stops the effect is emphasised as the (small) delay to the first vehicle and the (slight) early arrival of the second vehicle result in increasingly longer dwell times for the first bus and increasingly shorter dwell times for the second bus. Among the first studies describing the bus bunching effect is the seminal work by Newell and Potts (1964). They studied an idealised corridor with evenly spaced bus stops, identical travel times between stops, and constant passenger loads at bus stops. Given a small delay to the first bus of a service at a stop, Newell and Potts provide an analytical formulation of the deviation of bus arrival time to schedule for all buses and at all subsequent stops. They show that adjacent buses alternate between being behind and ahead of schedule, leading to bus bunching. The scale of the bunching effect and the stability of the bus system is affected not only by the size of the original delay to the first bus, but also by the ratio between passenger arriving rate and boarding rate referred to as the $k$ ratio. They show that if $1 / 2<k<1$, instability occurs. In 
practice, however, one would expect the passenger arrival rate to be smaller than the loading rate, i.e. $0<k<1 / 2$. In this case, Newell and Potts show that the downstream stops can recover from the original perturbation and return to schedule. Furthermore, bus bunching is more noted in high frequency services, where the headway between buses is small and the delay to headway ratio is larger than the threshold so that bus bunching amplifies (rather than being damped and remaining localised) further down the route.

Following on from Newell and Potts' work, there has been a significant body of literature designing operational strategies to avoid the bunching effect. In particular holding strategies of early buses as well as strategies to keep minimum distances between subsequent services have been analysed and shown to be successfully applied in literature. The holding strategies are implemented through building slacks in the schedule at key timing points and holding buses at these points to keep them to schedule (e.g. Osuna and Newell (1972); Newell (1974); Hickman (2001); Eberlein (2001); Cats et al (2012)). Due to the complexity of the problem, most of these early studies involve solving just one controlled timing point. Using a simulation approach, Hickman (2001) derived a set of static holding solutions, which do not respond to dynamic changes in the actual bus performances on the day. Eberlein et al (2001) proposed a model for dynamic bus holding which take real-time information on bus headways into consideration and strives to minimise passenger waiting time.

Daganzo (2009) explored a more systematic approach to the dynamic holding problem with real-time bus performance. Daganzo's method is able to consider holding at multiple timing points, therefore providing opportunity for return to schedule for long bus routes. In addition, the model takes into account random effects in bus travel time, bus dwell time and passenger demand, making it resemble better real-life situations. Daganzo and Pilachowski (2011) proposed an adaptive bus control scheme based on a two-way bus-to-bus cooperation, where a bus adjusts its speed to both its front and rear headways. They show that the scheme yields significant improvements in bus headways and bus travel time. Moving away from the traditional idea of schedule-adherence and achieving a target headway, Pilachowski (2009) proposed to use GPS data to counteract directly the cause of the bunching by allowing the buses to cooperate with each other and to determine their speed based on relative position, while Bartholdi and Eisenstein (2012) proposed a self-coordinating method to equalise bus headway.

Despite these recent developments, most of the existing studies present an oversimplified model of the bus bunching phenomenon, notably with a single line of service, with fixed service 
frequency, uniformly distributed (in time and space) passenger flows, and no bus overtaking. They neglect important aspects of real-life bus systems, such as passenger behaviour, en-route service perturbation, transport operator policies such as holding and overtaking, and complex network features such as common lines. Newell and Potts (1964), for instance, assume fixed frequency, constant dwell times, equal-distance stops and equal-travel time between stops, and that buses cannot overtake. In real-life situations, busy urban corridors are often served by multiple lines of bus services, with different frequencies and different sequence of stops. Besides, when buses are bunched at the stop, some passengers are likely to stick to the front bus, while some others prefer to get to the back bus, especially if there is a chance that it overtakes the front one. Consideration of this queuing behaviour will hence impact the dwell time of buses and the order of bus departures.

Exploring the effect of route sections served by multiple buses, Hernández et al (2015) proposed real-time control strategies for a corridor with multiple bus services where the common section is short. Schmöcker et al (2016) formulate the bunching problem for common line sections including overtaking between lines, and found that common lines can contribute to dampen bus bunching when overtaking is allowed for. Their model is simplified though by ignoring capacity constraints and the alighting process which makes it not yet practice ready.

In addition to bus line operation, it is also essential to pay attention to the bus stop operation when analyzing the bunching problem, because stops are usually the main bottleneck as discussed by Fernández (2010). Fernández defined the stop mechanism clearly and distinguish one-platform and divided-platform stops, where each platform contains one or several berths to load passengers. A simulation approach involving a passenger module, bus arrival and departure modules is then developed to assess the performance of the bus stop by passenger waiting time, bus delays and bus queue length. Similarly, Gu et al (2011) investigated the relationship between number of berths to load the passengers at one stop and bus headway or bus dwelling time. An approach to optimize the number of berths according to boarding demand is proposed in their work. It should be noted though that in their work as well as in Fernández (2010) overtaking is not allowed, even under the multiple-berth cases. As we will discuss in this paper, overtaking policy has the potential though to be an effective way to reduce bunching.

Given these gaps in the literature, in this paper, we focus on the passenger behaviour when there is more than one bus stopping at the stop at the same time. We investigate especially their 
choices and possible switching actions from the queue of the bus they are waiting to board to that of the coming one. A parameter $\gamma$ is introduced to denote the percentage of passengers remaining in queue for the front bus. We presume the same percentage applies further to passengers arriving at the stop during the dwell time, $\gamma$ thus could be regarded as the general "front-bus preference". Different scenarios of arrival and departure sequences are discussed respectively. Furthermore, we consider resulting differences in bunching depending on whether overtaking of buses at bus stops is allowed or not. In contrast to Fernández (2010) and Gu et al (2011) we hence model the propagation of delays along a corridor.

Section 2 of the paper sets out the basic model notation. Section 3 describes the bus propagation model, without and with bus overtaking. The critical point of the propagation model is obtaining the dwell time at stops. This is described in Section 4 where we develop a formulation to obtain the number of passengers who board the front bus when buses are bunched. Four evaluation indices of the system performance under different front-bus preference scenarios are then proposed in Section 5, and the performance of the model is illustrated through case studies in Section 6. Finally, Section 7 concludes the study and discusses the implications on network design.

\section{Notation and Basic Assumptions}

The following notation will be used throughout the paper.

Let

$m \quad$ bus number according to arrival time at the bus stop with $m=0,1,2, \ldots, \mathrm{M}$

$n \quad$ bus stop number with $n=0,1,2, . . \mathrm{N}$

$h \quad$ headway of the line

The above set of variables defines the basic service characteristics. Next we introduce bus and stop specific variables:

$a_{m, n} \quad$ time at which bus $m$ arrives at stop $n$

$d_{m, n} \quad$ time at which bus $m$ leaves stop $n$

$v_{m, n} \quad$ travel time of bus $m$ between stops $n-1$ and $n$; taken as fixed value in this study

$\rho_{m, n} \quad$ initial "exogenous" delay to bus $m$ before or at the $n$th stop

$\Delta_{m, n} \quad$ passenger arrival period over which demand for bus $m$ at stop $n$ accumulates 
$\bar{\Delta}_{m, n} \quad$ scheduled passenger arrival period over which demand for bus $m$ at stop $n$ accumulates

$w_{m, n} \quad$ dwell time of bus $m$ at stop $n$

$b_{m} \quad$ passenger loading rate of bus $m$

$q_{n} \quad$ passenger arriving rate at stop $n$ for passengers

$k_{m, n} \quad$ ratio between passenger arriving and loading rate for bus $m$ at stop $n$

Finally, we introduce two variables that are time and stop specific but not bus specific:

$\xi_{n}(t) \quad$ last departure of any bus from stop $n$ given time $t$

$x_{n}(t) \quad$ queue of passengers at stop $n$ at time $t$ who want to board

Both $a_{m, n}$ and $d_{m, n}$ are measured as absolute values of time passed since the first bus leaves the terminal/ enters the corridor of interest. We note that $m$ does not necessarily have to be in the dispatching order from depot if we allow for overtaking. If the leading bus has to board a large amount of passengers the subsequent bus the subsequent bus might overtake it if permitted and hence the arrival order at the subsequent stop reverses. We assume that bus travel time between stops is constant so that $v_{m, n}$ simplifies to $v$. This is though not a restrictive assumption as we assume that buses can be subject to random, "exogenous" delays at stops denoted by $\rho_{m, n}$. For illustration purposes in the case studies we assume that one delay occurs at a bus stop in the beginning of the modelled section. This event triggers the subsequent bunching effect. Boarding door is presumed to be open during exogenous delay in which newly coming passengers can keep boarding. If the entrance door is assumed closed, passengers accumulated in the delay should be distributed to next bus. Instead of delays at stops one could also introduce delays on links. The difference between assuming random link travel times and delays at stops is that in the latter passengers arriving at the stop during the delay period can board the bus whereas in the former obviously they cannot. Replacing stop delays $\rho_{m, n}$ by one (or multiple) link delays presents no methodological difficulty in the approach presented hereafter.

The boarding time per passenger is primarily depending on doors and ticketing system. Sun et al (2014) report that the loading time per passenger further depends on the interaction between boarding and alighting passengers. In the following we omit this issue and instead make the simplifying assumption that all buses are identical, i.e. have the same boarding rate per passenger, so that we can assume a fixed $b_{m}$ and omit the subscript $m$. Further, whereas 
Fonzone et al (2015) assume that arrival patterns are time dependent here we assume a constant $q_{n}$. With these assumptions also $k$ becomes time and bus independent and can be defined as

$k_{n}=\frac{q_{n}}{b}$

Clearly, to avoid queues at bus stops building up over the analysis period, we require

$0 \leq k_{n}<1$

The passenger arrival period, $\Delta_{m, n}$, for a regular service will be equal to the service headway. In case of a bunched service, various definitions are possible, depending on bus stop layout, operational policy as well as passenger behaviour. For example, if control staff is at the bus stop the operator might restrict the boarding of newly arriving passengers while the bus is already loading passengers. In an uncontrolled system passengers arriving while two buses are at the same time at the stop will have a choice between these.

In this paper, passengers who arrive during the dwell time can still board the bus, which means the arrival period of passengers is not equal to headway which is the interval of two adjacent arrivals, but is defined as the interval of two adjacent departures. $\Delta_{m, n}$ can be generally obtained as

$\Delta_{m, n}=\min \left\{d_{m, n}-d_{m \prime, n} \mid d_{m \prime, n} \leq d_{m, n}\right\}, \forall m, n$

which simplifies to (4) if overtaking is not allowed or does not occur

$\Delta_{m, n}=d_{m, n}-d_{m-1, n}$

We further note that equation (5) to obtain dwell times does not hold if several buses are serving the stop. We elaborate on this in the Section 4.

$w_{m, n}=\Delta_{m, n} k_{n}$ 


\section{Bus Propagation Model}

Bus services are propagated by four primary continuous events which are referred to as arrival at a stop, dwelling at the stop, departure from the stop and travel to next stop. The arrival and departure are two time points while correspondingly dwell and travel are events over a period of time during which delays might occur. In line with previous notation we utilise $a_{m-1, n}$, $a_{m+1, n}$ and $d_{m-1, n}$ to describe arrivals and departures of previous and subsequent buses relative to bus $m$. The departure time can be obtained with (6), and the arrival of the same bus at the subsequent stop with (7). As noted the travel time between stops is not the focus of this paper and simply presumed to be constant. This leaves us with obtaining the duration of the fourth event, the dwell time, which is developed in Section 4.

Below is a basic bus propagation algorithm to solve the problem. The algorithm is recursive in that it obtains the time point and time duration of the four events for each stop and each bus in order of arrivals at the stops. As it is presumed that downstream stops have no influence on dwell time at upstream stops we can solve the algorithm in increasing order of stops from the terminal. Once all departure times from a stop are known events at the downstream stop can be calculated.

In line with our discussion in previous section we note that perturbations to the system arise through random delays during the dwell time process which then might trigger a series of bunching events as our case study will show.

Bus Propagation Algorithm

Initialisation

Set $a_{m, 1} \forall m$

Set $\Delta_{1, n} \forall n$

For each stop $n$ in increasing order

Sort buses according to arrival times at stop

For each bus $m$ in order of increasing arrival times obtain

$$
\begin{aligned}
& \text { Obtain } w_{m, n} \text { as in Section } 4 \\
& d_{m, n}=a_{m, n}+w_{m, n}+\rho_{m, n}
\end{aligned}
$$




$$
a_{m, n+1}=d_{m, n}+v_{m, n}
$$

\section{Passenger Choice and Resulting Dwell Times for Bunched Buses}

\subsection{Possible Event Sequences at the Bus Stop}

In case there is no service disturbance and only one bus at a time serves a bus stop obtaining the dwell time is straightforward by integrating passenger arrivals over the time period for which passengers accumulate for a specific bus. This is described in "Case A" and Equation (8) further below.

Let us now consider the case that two buses are boarding passengers at the same time. As described in the introduction $\gamma$ denotes the front-bus preference of passengers waiting to board and newly arriving during the dwell time of the buses. Therefore, with $\gamma=1$ all passengers keep boarding the front bus, whereas with $\gamma=0$ all passengers at the bus stop swap to board the bus that arrived later.

Considering departure of the previous bus and arrival of the bus subsequent to $m$, each column of Table 1 shows a possible event sequence. In the top row the number stands for the number of buses at the bus stop and the letter behind the number for the position of the bus of interest at the bus stops. In other words, " $2 \mathrm{f}$ " stands for the bus being the front bus of two at the stop, " $2 \mathrm{~b}$ " for the bus being at the back of two buses at the bus stop and " $3 \mathrm{~m}$ " for the bus being the middle one of three at the bus stop. Similarly, " $3 b$ " denotes the last bus in three buses. The arrow stands for the state transition of the bus due to arrivals or departures of other buses.

Table 1 is accompanied by an illustration of all the event sequences as in Figure 1. The box in dotted line is to demarcate the berth area. $a_{m}$ stands for the time point at which bus $m$ enters into the berth. $d_{m}$ denotes the time point when bus $m$ is leaving the stop.

The lower part of Table 1 then provides an overview on the solutions that need to be obtained separately depending on $\gamma$ and depending on whether we allow for overtaking between buses or not. Case 1 denotes the above-mentioned non-bunched case in that bus $m$ arrives after the previous bus has left and departs before the next bus has arrived. The case clearly can occur for all $\gamma$ and independent of whether overtaking is considered or not. It can be solved with the equations shown under Section A.

The second case, $1 \rightarrow 2 \mathrm{f}$, denotes the case that while the bus is still boarding the subsequent 
bus arrives. In this case, and all subsequent cases, hence the solution depends on $\gamma$. Firstly if $\gamma=1$ then all passengers board the bus of interest, so that the waiting time of the bus is identical to the case without considering bus $m+1$. The case also makes bunching worse compared to smaller $\gamma$ values, as bus $m+1$ does not help to relieve bus $m$ though it has already caught up with this bus. For cases $1>\gamma>0$ the relief by bus $m$ needs to be taken into account as shown in Section B. With decreasing $\gamma$ the dwell time of bus $m$ will continuously decrease so that in the extreme case of $\gamma=0$ bus $m$ leaves immediately when bus $m+1$ is arriving. This corresponds to the case of the bus driver in the front bus trying to reduce the bunching effect by pushing all passengers to the back bus.

In case overtaking is allowed and if $\gamma>0.5$ bus $m+1$ will overtake bus $m$ as this means more than half the passengers will remain boarding bus $m$. In case of $\gamma=0.5$ the buses become "twin buses" as they depart at the same time, whereas in the case of $\gamma<0.5$ bus $m$ will depart before bus $m+1$.

We note that obtaining the exact dwell time in case of $1>\gamma>0.5$ is not possible with our analytical solution approach. Bus $m+1$ will overtake bus $m$ so that from $d_{m+1}$ until $d_{m}$ bus $m$ becomes the only bus at the stop again. Hence obtaining $d_{m+1}$ is required in order to obtain $d_{m}$. For this case we linearly approximate the dwell time from the limiting cases $\gamma=1$ and $\gamma=0.5$ which we can solve directly. The dwell time for bus $m$ must be smaller than for $\gamma=1$ but larger than for $\gamma=0.5$.

The following five columns all presume that bus $m$ arrives while bus $m-1$ has not yet departed. Firstly consider a system without overtaking. For $\gamma \geq 0.5$ this hence means that bus $m$ leaves together with bus $m-1$ so that the behaviour of bus $m+1$ does not have to be considered and one always obtains $w_{m}=d_{m-1}-a_{m}-\rho_{m}$ which is equivalent to $d_{m}=d_{m-1}$. In case of $\gamma<$ 0.5 instead bus $m$ will have to pick up more than half the passengers queuing at the stop and hence these passengers need to be considered in determining the dwell time of bus $m$ (Cases C and $\mathrm{D}$ in the table). We assume that only two buses can board passengers at the same time, i.e. a third (and fourth etc.) bus that might be at the stop at the same time cannot pick up passengers until one of the front two buses has departed. Under this assumption case $2 b \rightarrow 3 m \rightarrow 2 f$ simplifies to the $2 \mathrm{~b} \rightarrow 2 \mathrm{f}$ case. That is, until departure of bus $m-1$, bus $m$ is the latter of two buses. At departure of bus $m-1$ then bus $m$ becomes the front bus of two boarding buses. This case is denoted as F and solved below. Note further that for $\gamma=0$ again the solutions simplify. Whenever the bus transits into the $2 \mathrm{f}$ state, it can leave immediately, if it is in the $2 \mathrm{~b}$ state, bus 
$m-1$ can leave immediately and hence it becomes identical to the 1 bus waiting time if bus $m$ can leave before bus $m+1$ is arriving.

We suggest the assumption that at most 2 buses can simultaneously load passengers is realistic in most cases. It further eases the complexity of the multiple bus bunching problem. In case 3 or more buses are bunched at the stop, the third (and fourth, fifth..) bus following it are prevented to enter the berth to pick-up passengers until space becomes available. This means cases of four or more bunched buses are also covered within our formulation where the bus has to wait until it becomes bus $3 \mathrm{~b}$ thus entering the right most scenario in Table 1 . Note further, that in fact any bunching of 3 or more buses to be a 2-bus bunching. Taking the $3 \mathrm{~b}$ case, bus $m$ is bunched after two leading buses and overtaking is permitted, it is supposed to depart instantly after finishing unloading passengers, and $w_{m}$ is considered 0 here. If overtaking is prohibited, it always leaves together with the two previous buses for $\gamma \geq 0.5$ and it is converted into $2 b \rightarrow 1$ case for $\gamma<0.5$. Finally, note that for $\gamma<0.5$ no overtaking occurs at the bus stop as the previous bus will always be able to leave before the subsequent bus as it will have to pickup less passengers of the remaining queuing travellers at the stop.

The remainder of this chapter obtains the formulation for the analytical solution to the cases discussed above. 
Table 1 Possible event sequences from the viewpoint of bus $m$ and corresponding calculation of dwell time

\begin{tabular}{|c|c|c|c|c|c|c|c|c|}
\hline \multicolumn{9}{|c|}{ Possible Event Sequences } \\
\hline Case & 1 & $\mathbf{1} \rightarrow \mathbf{2 f}$ & $2 b \rightarrow 1$ & $\mathbf{2 b} \rightarrow \mathbf{1} \rightarrow \mathbf{2 f}$ & $\mathbf{2 b}$ & $2 \mathrm{~b} \rightarrow 3 \mathrm{~m} \rightarrow 2 \mathrm{f}$ & $2 \mathrm{~b} \rightarrow 3 \mathrm{~m}$ & $3 \mathrm{~b}(\rightarrow 1)$ \\
\hline \multirow{4}{*}{$\begin{array}{l}\text { event } \\
\text { sequence } \\
\text { (increasing } \\
\quad \text { time) }\end{array}$} & & \multicolumn{6}{|c|}{$a_{m}$} \\
\hline & \multicolumn{2}{|r|}{$a_{m}$} & \multicolumn{2}{|c|}{$d_{m-1}$} & $d_{m}$ & \multicolumn{2}{|c|}{$a_{m+1}$} & \multirow{3}{*}{$\begin{array}{c}d_{m-2} \\
d_{m-1} \\
d_{m} \\
\end{array}$} \\
\hline & $d_{m}$ & $a_{m+1}$ & $d_{m}$ & $a_{m+1}$ & \multirow{2}{*}{$\begin{array}{l}a_{m+1} \\
d_{m-1}\end{array}$} & $d_{m-1}$ & $d_{m}$ & \\
\hline & $a_{m+1}$ & $d_{m}$ & $a_{m+1}$ & $d_{m}$ & & $d_{m}$ & $d_{m-1}$ & \\
\hline \multicolumn{9}{|c|}{ Solution to Specific Cases } \\
\hline \multicolumn{9}{|l|}{ No Overtaking } \\
\hline$\gamma=1$ & \multirow[t]{5}{*}{$\mathrm{A}$} & $\begin{array}{l}\text { A }(m+1 \text { waits } \\
\text { behind } m)\end{array}$ & \multirow{3}{*}{$\begin{array}{l}\quad w_{m} \\
=d_{m-1} \\
-a_{m} \\
\text { (m-1 and } m \\
\text { bunched) }\end{array}$} & \multirow{3}{*}{$\begin{array}{l}\text { Does } \\
\text { occur: } \\
d_{m}=a_{m+1} \\
=d_{m-1}\end{array}$} & \multirow{5}{*}{$\begin{array}{l}\text { Does not } \\
\text { occur: case } \\
\text { presumes } \\
\text { overtaking }\end{array}$} & \multirow{3}{*}{$\begin{array}{l}w_{m} \\
=d_{m-1}-a_{m} \\
(m-1 \text { and } m \\
\text { bunched })^{*}\end{array}$} & \multirow{5}{*}{$\begin{array}{l}\text { Does not } \\
\text { occur: case } \\
\text { presumes } \\
\text { overtaking }\end{array}$} & \multirow{3}{*}{$\begin{array}{l}w_{m} \\
=d_{m-2}-a_{m} \\
=d_{m-1}-a_{m}\end{array}$} \\
\hline $1>\gamma>0.5$ & & B $\quad(m \quad$ and & & & & & & \\
\hline$\gamma=0.5$ & & $\begin{array}{l}m+1 \\
\text { bunched) }\end{array}$ & & & & & & \\
\hline $0.5>\gamma>0$ & & $\mathrm{~B}$ & \multirow[t]{2}{*}{$\mathrm{C}$} & $\mathrm{D}$ & & $\mathrm{F}^{*}$ & & \multirow[t]{2}{*}{$\mathrm{C}$} \\
\hline$\gamma=0$ & & $\begin{array}{l}w_{m} \\
=a_{m+1} \\
-a_{m}\end{array}$ & & $\begin{array}{l}w_{m} \\
=a_{m+1} \\
-a_{m}\end{array}$ & & $\begin{array}{l}w_{m}=d_{m-1}- \\
a_{m} *\end{array}$ & & \\
\hline \multicolumn{9}{|l|}{ Overtaking } \\
\hline$\gamma=1$ & \multirow[t]{3}{*}{$\mathrm{A}$} & $\begin{array}{l}\text { A } \quad(m+1 \\
\text { leaves } \\
\text { immediately) }\end{array}$ & \multirow[t]{2}{*}{$\begin{array}{l}\text { Does not } \\
\text { occur: } \\
d_{m}<d_{m-1}\end{array}$} & \multirow[t]{2}{*}{$\begin{array}{c}\text { Does not } \\
\text { occur: } w_{m}= \\
0 \text { and } \\
d_{m}<d_{m-1}\end{array}$} & $\begin{array}{c}w_{m}=0 \\
\quad \text { and } \\
\text { overtaking) }\end{array}$ & \multirow[t]{2}{*}{$\begin{array}{c}\text { Does not } \\
\text { occur: } w_{m}= \\
0 \text { and } \\
d_{m}<d_{m-1}\end{array}$} & $\begin{array}{c}w_{m}=0(\text { and } \\
\text { overtaking; } \\
\text { only if } \\
\left.a_{m}=a_{m+1}\right)\end{array}$ & \multirow[t]{3}{*}{$\begin{array}{c}w_{m}=0 \\
\text { (quick } \\
\text { unloading and } \\
\text { overtaking) }\end{array}$} \\
\hline $1>\gamma>0.5$ & & $\begin{array}{l}\mathrm{X} \quad(m+1 \\
\text { overtakes } m) \\
\end{array}$ & & & $\mathrm{E}$ & & $\mathrm{E}^{*}$ & \\
\hline$\gamma \leq 0.5$ & & \multicolumn{6}{|c|}{ Overtaking does not occur, identical to no overtaking case } & \\
\hline
\end{tabular}




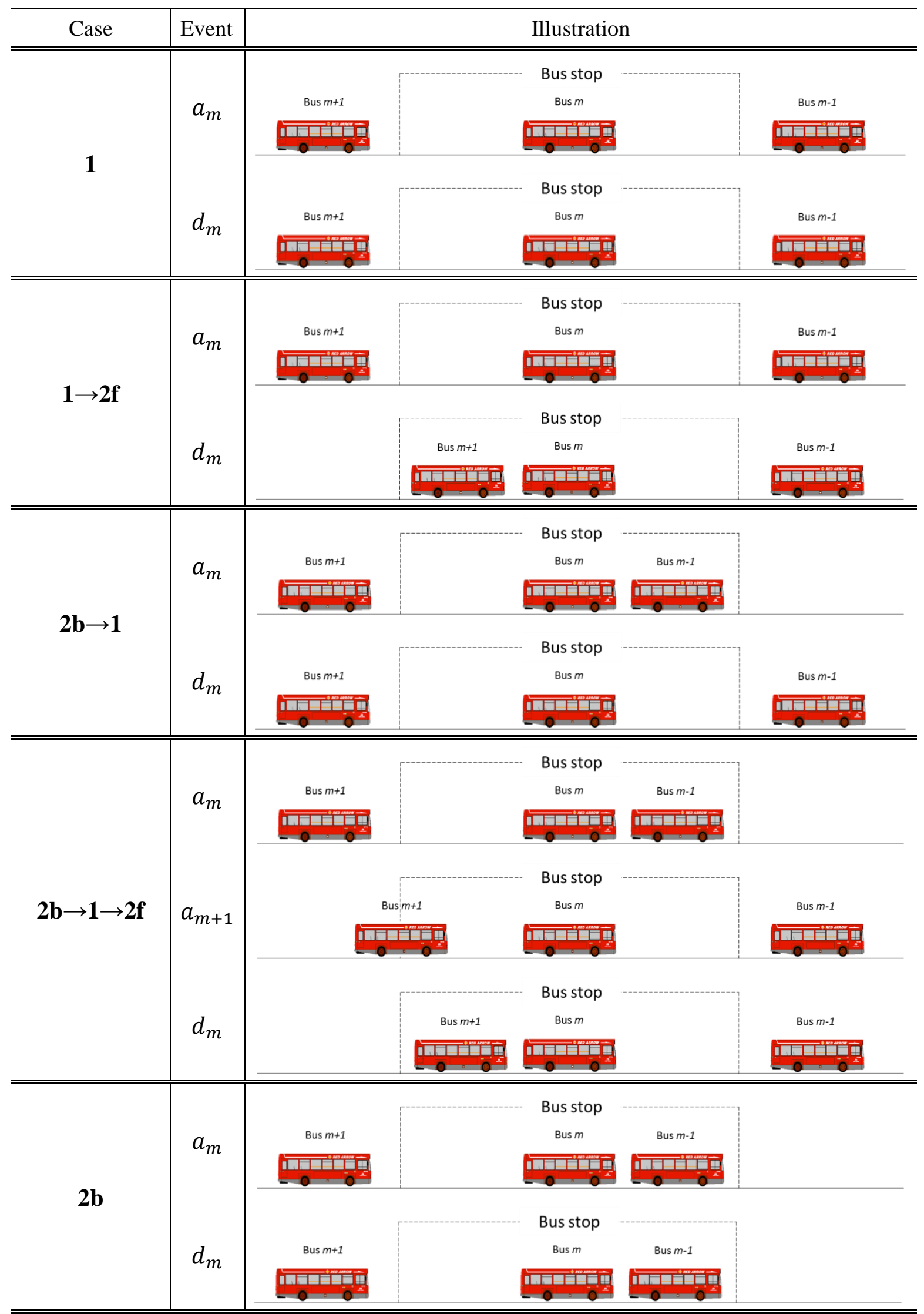




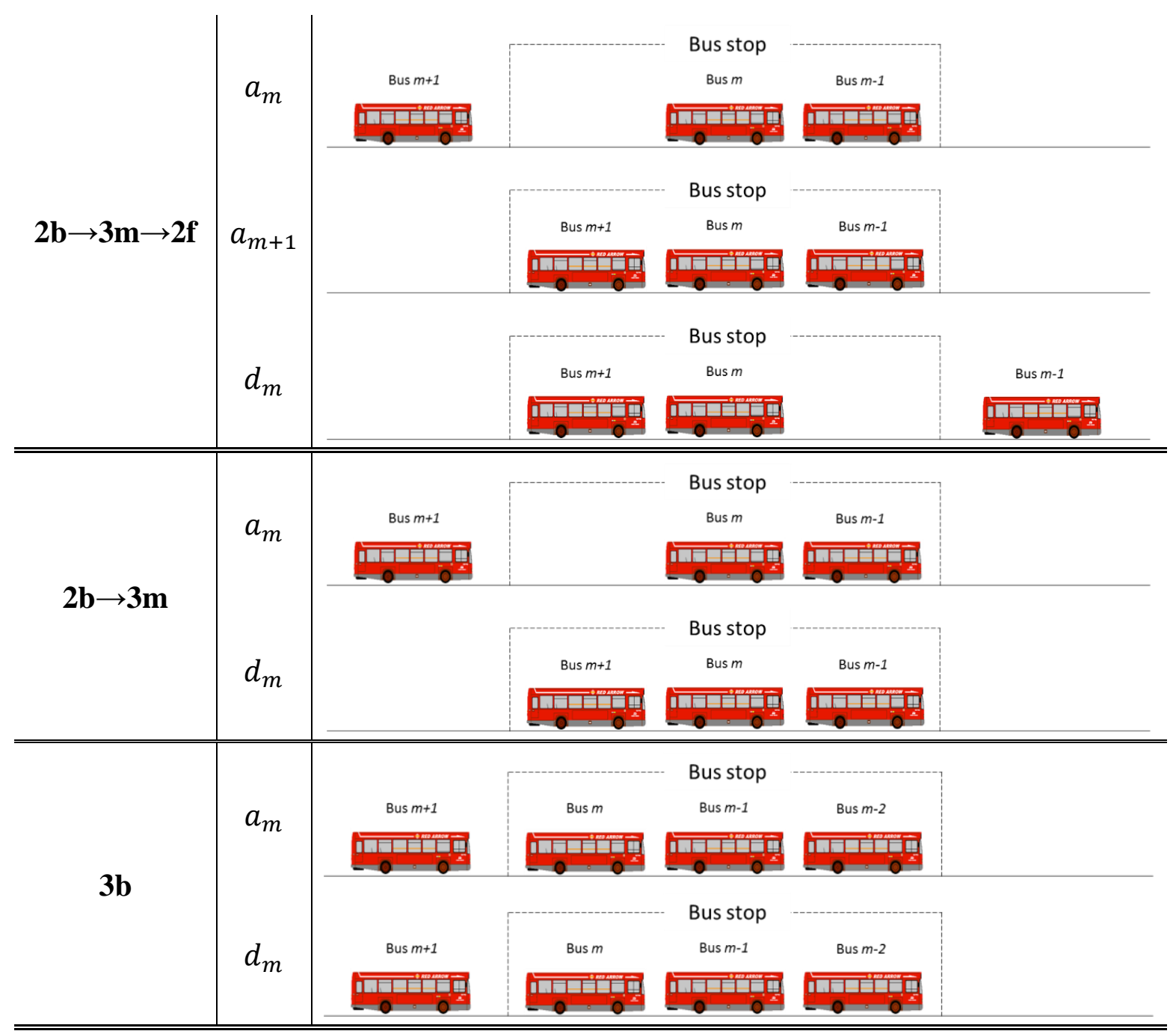

Figure 1 Event sequences from the viewpoint of bus $m$

\subsection{Waiting formulation for specific cases}

\section{$\underline{\text { A: 1bus, no bunching case }}$}

$w_{m, n}=\frac{1}{b} \int_{d_{m-1, n}}^{d_{m, n}} q_{n} d t=\frac{1}{b} \int_{d_{m-1, n}}^{a_{m, n}+w_{m, n}} q_{n} d t=k_{l n}\left(a_{m, n}+w_{m, n}+\rho_{m, n}-d_{m-1, n}\right)$

$w_{m, n}=\frac{k_{l n}\left(a_{m, n}+\rho_{m, n}-d_{m-1, n}\right)}{\left(1-k_{l n}\right)}$

$\underline{\text { B: } 1 \rightarrow 2 f \text { and assuming no overtaking (either because } \gamma<0.5 \text { or because overtaking not }}$ allowed)

We obtain the queue $x$ at stop $n$ at time $a_{m+1, n}$ as

$x_{n}\left(a_{m+1, n}\right)=q\left(a_{m+1, n}-\xi_{n}\left(a_{m+1, n}\right)\right)-b\left(a_{m+1, n}-a_{m, n}\right)$ 
Waiting time of bus $m$ can be obtained as

$w_{m, n}=\left(a_{m+1, n}-a_{m, n}\right)+\frac{\gamma}{b}\left(x_{n}\left(a_{m+1, n}\right)+\int_{a_{m+1, n}}^{d_{m, n}} q_{n} d t\right)$

Which is equivalent to

$w_{m, n}=\left(a_{m+1, n}-a_{m, n}\right)+\frac{\gamma x_{n}\left(a_{m+1, n}\right)}{b(1-\gamma k)}+\frac{\gamma k}{1-\gamma k} \rho_{m, n}$

The passengers left behind by bus $m$ is obtained as

$x_{n}\left(d_{m, n}\right)=x_{n}\left(a_{m+1, n}\right)+\int_{a_{m+1, n}}^{d_{m, n}}\left(q_{n}-2 b\right) d t$

\section{$\underline{C: 2 b \rightarrow 1 \text { and no overtaking because } y<0.5}$}

We firstly obtain the queue of passengers at the stop when bus $m$ is arriving as

$x_{n}\left(a_{m, n}\right)=q\left(a_{m, n}-\xi_{n}\left(a_{m, n}\right)\right)-b\left(a_{m, n}-a_{m-1, n}\right)+x_{n}\left(\xi_{n}\left(a_{m, n}\right)\right)$

The passengers left behind by the bus previously departed are emphasized as $x_{n}\left(d_{m-1, n}\right)$ and distributed to bus $m$

The waiting time can be formulated as follows

$w_{m, n}=\frac{1-\gamma}{b}\left(x_{n}\left(a_{m, n}\right)+\int_{a_{m, n}}^{d_{m-1, n}} q_{l n} d t\right)+\frac{1}{b} \int_{d_{m-1, n}}^{d_{m, n}} q_{l n} d t$

And hence

$w_{m, n}=\frac{(1-\gamma) x_{n}+b \gamma k\left(a_{m, n}-d_{m-1, n}\right)+b k \rho_{m, n}}{b(1-k)}$

\section{$\underline{D:} \mathbf{2 b} \rightarrow \mathbf{1} \rightarrow \mathbf{2 f}$ and no overtaking because $\gamma<0.5$}

In this case we need to obtain the queue of passengers at the arrival of bus $m$ (when bus $m$ enters the $2 \mathrm{~b}$ state) as well as at time $a_{m+1, n}$ when bus $m$ enters the $2 \mathrm{f}$ state. In fact, as shown below the $x_{n}\left(a_{m+1, n}\right)$ obviously depends on $x_{n}\left(a_{m, n}\right)$.

$x_{n}\left(a_{m, n}\right)=q_{n}\left(a_{m, n}-\xi_{n}\left(a_{m, n}\right)\right)-b\left(a_{m, n}-a_{m-1, n}\right)+x_{n}\left(\xi_{n}\left(a_{m, n}\right)\right)$

$x_{n}\left(a_{m+1, n}\right)=x_{n}\left(a_{m, n}\right)-2 b\left(d_{m-1, n}-a_{m, n}\right)-b\left(a_{m+1, n}-d_{m-1, n}\right)+q_{n}\left(a_{m+1, n}-a_{m, n}\right)$ 
As this case implies that the bus is still at the stop at the arrival time of bus $m+1$ the waiting time can be obtained as

$w_{m, n}=\left(a_{m+1, n}-a_{m, n}\right)+\frac{\gamma}{b}\left(x_{n}\left(a_{m+1, n}\right)+\int_{a_{m+1, n}}^{d_{m, n}} q_{n} d t\right)$

Which can be solved to

$w_{m, n}=\left(a_{m+1, n}-a_{m, n}\right)+\frac{\gamma x_{n}\left(a_{m+1, n}\right)}{b(1-\gamma k)}+\frac{\gamma k}{1-\gamma k} \rho_{m, n}$

The passengers left behind by bus $m$ is obtained as

$x_{n}\left(d_{m, n}\right)=x_{n}\left(a_{m+1, n}\right)+\int_{a_{m+1, n}}^{d_{m, n}}\left(q_{n}-2 b\right) d t$

\section{E: $2 b$ (only for $\gamma>0.5$, includes overtaking)}

We obtain again the queue at the stop when bus $m$ is entering stage $2 b$ as

$x_{n}\left(a_{m, n}\right)=q\left(a_{m, n}-\xi_{n}\left(a_{m, n}\right)\right)-b\left(a_{m, n}-a_{m-1, n}\right)$

This lead to

$w_{m, n}=\frac{1-\gamma}{b}\left(x_{n}\left(a_{m, n}\right)+\int_{a_{m, n}}^{d_{m, n}} q_{n} d t\right)$

And hence

$w_{m, n}=\frac{(1-\gamma)\left(x_{n}\left(a_{m, n}\right)+b k \rho_{m, n}\right)}{b(1-k+\gamma k)}$

The passengers left behind when bus $m$ departures is obtained as

$x_{n}\left(d_{m, n}\right)=x_{n}\left(a_{m, n}\right)-2 b w_{m, n}+\int_{a_{m, n}}^{d_{m, n}} q_{n} d t$

\section{$\underline{F: 2 b \rightarrow(3 m) \rightarrow 2 f, ~ n o ~ o v e r t a k i n g ~(b e c a u s e ~} \gamma<0.5)$}

Finally, with our assumption that only two buses are boarding passengers simultaneously, we obtain that in this case the bus transfer immediately from the $2 \mathrm{~b}$ state into the $2 \mathrm{f}$ state. The transition occurs at time $d_{m-1, n}$ and we obtain the queue at this point in time by

$x_{n}\left(a_{m, n}\right)=q\left(a_{m, n}-\xi_{n}\left(a_{m, n}\right)\right)-b\left(a_{m, n}-a_{m-1, n}\right)+x_{n}\left(\xi_{n}\left(a_{m, n}\right)\right)$

$x_{n}\left(d_{m-1, n}\right)=x_{n}\left(a_{m, n}\right)-2 b\left(d_{m-1, n}-a_{m, n}\right)$ 


$$
=q\left(a_{m, n}-\xi_{n}\left(a_{m n}\right)\right)+b\left(a_{m, n}+a_{m-1, n}-2 d_{m-1, n}\right)
$$

Then the waiting time can be obtained by

$w_{m, n}=\left(d_{m-1, n}-a_{m, n}\right)+\frac{\gamma}{b}\left(x_{n}\left(d_{m-1, n}\right)+\int_{d_{m-1, n}}^{d_{m, n}} q_{n} d t\right)$

And hence

$w_{m, n}=\left(d_{m-1, n}-a_{m, n}\right)+\frac{\chi_{n}\left(d_{m-1, n}\right)}{b(1-\gamma k)}+\frac{\gamma k}{1-\gamma k} \rho_{m, n}$

The passengers left behind by bus $m$ is obtained as

$x_{n}\left(d_{m, n}\right)=x_{n}\left(d_{m-1, n}\right)+\int_{d_{m-1, n}}^{d_{m, n}}\left(q_{n}-2 b\right) d t$

\section{$\underline{X: 1->2 f}, y>0.5$, with overtaking}

The only case that we can not solve accurately is the case denoted by $\mathrm{X}$ in above table. As noted the reason is that the departure time for bus $m+1$ needs to be known or solved simultaneously when we solve for the departure time of bus $m$. One could do so by a time step simulation approach similar to work described in Fonzone et al (18). However, as the two limiting cases for $\gamma=1$ (case A) and $\gamma=0.5$ (case B) can be solved accurately and since we know that the waiting time is continuously decreasing for bus $m$ in state $2 \mathrm{f}$ for decreasing $\gamma$ we can approximate:

$w_{m, n}=2((\gamma-0.5) A+(1-\gamma) B)$

\section{Evaluation Measures}

We assume high frequency service in which passengers arrive uniformly at the stop. In such situations headway evenness is a more effective index than punctuality as passengers will not mind which specific run of a service they board. Therefore our main service regularity index is the service interval duration $\Delta_{m, n}$ as in (4) and its standard deviation, which depend on the exogeneous and delay and our input parameter of interest $\gamma$. We note the difference to service headway deviation which is based in most literature on service arrivals. Instead, $\Delta_{m, n}$ has a direct effect on the waiting time of passengers at the stop as it includes the dwell time.

The mean and maximum of $\Delta_{m, n}$ of all the bus services can be obtained respectively as 
$\bar{W}=\frac{\sum_{n} \sum_{m} \Delta_{m, n}}{M \times N}$

$\widehat{w}=\max _{n} \max _{m} \Delta_{m, n}$

Further, the total standard deviation of $\Delta_{m, n}$ and the stop-specific maximum standard deviation of $\Delta_{m, n}$, can be obtained respectively as

$\bar{\sigma}=\sqrt{\frac{\sum_{n} \sum_{m}\left(\Delta_{m, n}-\bar{\Delta}_{m, n}\right)^{2}}{M \times N}}$

$\hat{\sigma}=\max _{n} \sqrt{\frac{\sum_{m}\left(\Delta_{m, n}-\bar{\Delta}_{m, n}\right)^{2}}{M}}$

Standard deviation is a system index and of significance to operators, whereas arguably the maximum waiting times are more important to passengers as the frustration due to long delays is not a linear function.

\section{Case Study}

\subsection{Specifications}

This case study is a numerical test based on the analytical model proposed above. We consider a single line with 10 stops. The bus line runs with a frequency of $h=6 \mathrm{~min}$ and we assume that the travel time between two adjacent stops takes a constant value of $3 \mathrm{~min}$. We further assume that an initial random delay occurs for the 2 nd bus or both the 2 nd and 3 rd bus at the 2 nd stop. This means that the first bus is unaffected and hence runs with the expected headways and encounters the same (expected) dwell times at the stop. The impact of initial delay on bus trajectories is also investigated by varying its size from a slight delay to a delay longer than headway. In Section 6.2 and 6.3, only the 2nd bus encounters an initial delay whose size is $2 \mathrm{~min}$ at the 2 nd stop, and in Section 6.4, consequences of different initial delays are discussed.

To evaluate the effect of different passenger behavior, we model bus bunching with $\gamma=0$, $0.1,0.2, \ldots, 1$. We also distinguish different overtaking policies, since we expect to observe differences for $\gamma>0.5$. Besides, we vary the $k$-ratio since larger arriving-to-loading ratios lead to longer dwell times so that large $k$-ratios can also help to illustrate the effects of interest. We 
note that in real cases k-ratios are often lower, e.g. Liu and Sinha (2004) in a case study of the City of York, UK, report a ratio of approximately 0.1, with 1 passenger per minute arriving and the boarding process requiring $6 \mathrm{sec}$ per passenger. Electronic payment will further reduce the time it takes for passengers to board. At large stations though and during peak times obviously the arrival rate might be significantly higher.

\subsection{Illustration of resulting bus trajectories}

Figures 2(a) to (c) shows the bus trajectories for 3 extreme cases considering a moderate $k$ ratio of $k=0.25$. Red and green are used only to better distinguish subsequent buses. The case that all the passengers choose to board the back bus is illustrated in Figure 2(a). The case that all passenger will stick to the front bus and the back bus hence can overtake is shown in Figure 2(b). Figure 2(c) instead illustrates the inefficient case that the back bus is not allowed to overtake the front one although no one is boarding the back one.

Comparing Figures 2(b) and 2(c), one can especially observe that the bus system will provide a service with shorter maximal departure intervals and smaller variation of departure intervals if overtaking is possible: $\widehat{w}$ and $\bar{\sigma}$ are reduced by $45 \%$ and $35 \%$ respectively. We therefore observe that allowing for overtaking is of necessity if passengers show no propensity to take the back bus and/or the layout of the stop means passenger are unlikely to swap queues and take the back bus.

Furthermore, with $\gamma=1$ we can observe in Figure 2(b) that once the buses are bunched, a pair of buses keep overtaking each other if overtaking is possible. If overtaking is not allowed, as in Figure 2(c), a more severe phenomenon is that the second bus is "lost" for the system once the second bus has caught up with the former one; namely, the latter bus fails to pick up passengers when passengers always board the first bus and overtaking is not allowed, therefore the second bus ends up leaving and reaching the stops immediately after the former one.

Accordingly less disorder and better efficiency is illustrated in the case of $\gamma=0$ (Figure 2(a)). The front bus always leave instantly when the back bus arrives so that the front bus can pull away and reduce the bunching effect. We remind that in this study we assume deterministic passenger arrivals and the $k$ level illustrated in Figure 1 implies that the subsequent bus catches up with the front bus at the next stop again. In case of stochastic arrivals, we suggest that high back bus preference will be of even more benefit as the front bus might be able to create a 
distance between itself and the subsequent bus at least for some stops.

We notice further that the indices indicating service performances in Figures 2(a) and 2(b) are almost the same. Therefore, we suggest that when overtaking is not a feasible option for the service (possibly because of narrow roads) the system does not lose much efficiency as long as the passengers can be persuaded to use the back bus of two boarding at the same time. 


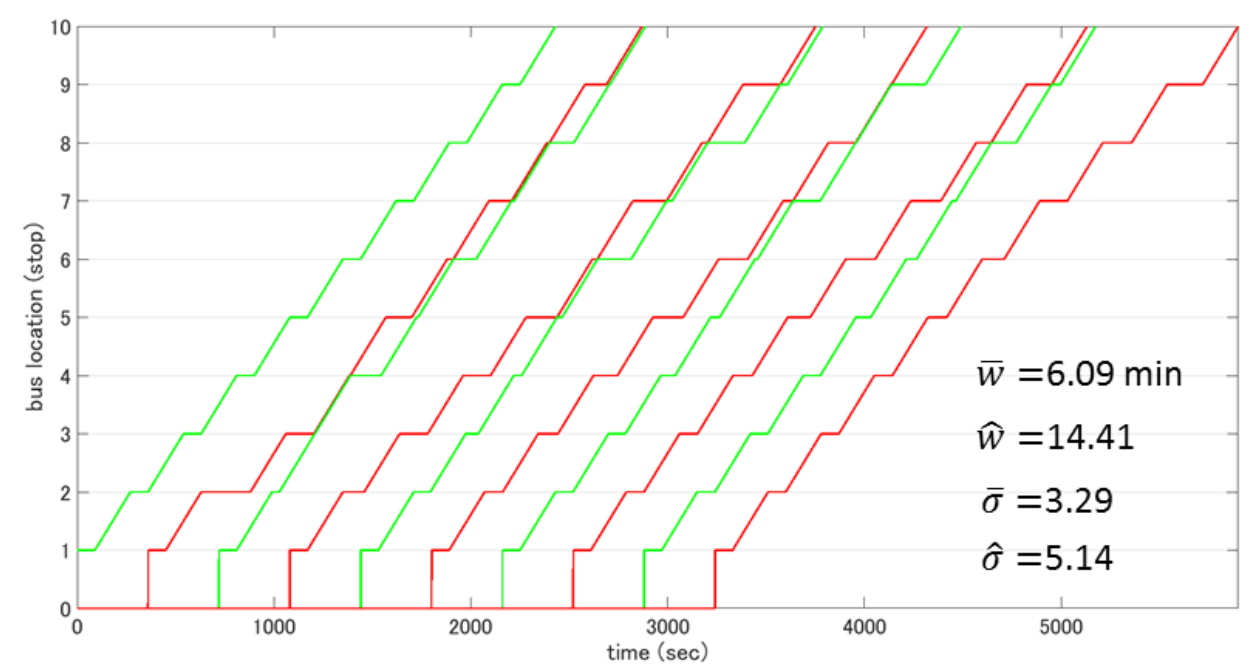

(a) $\gamma=0$ (overtaking will not occur)

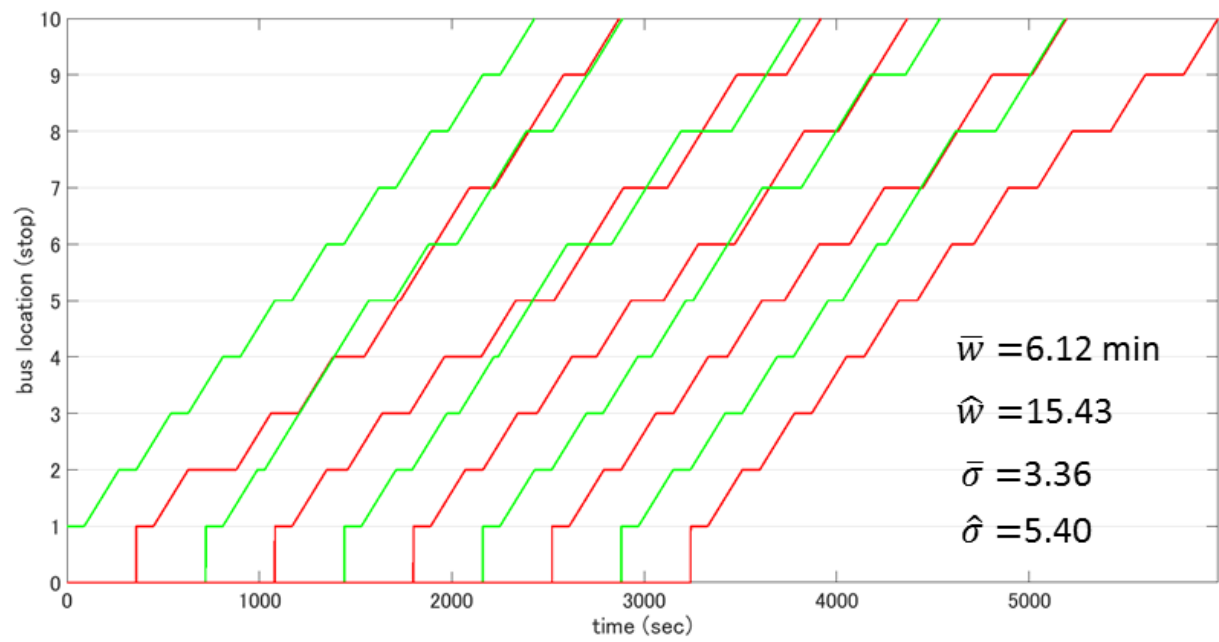

(b) $\gamma=1$ with overtaking allowed

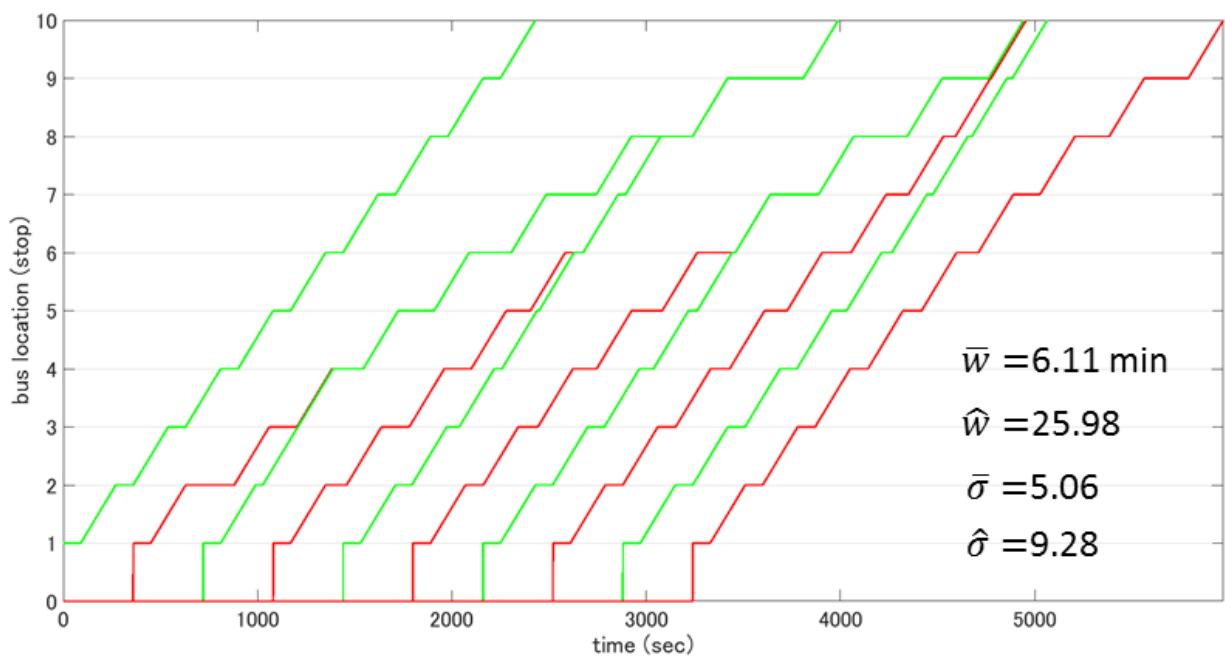

(c) $\gamma=1$ without overtaking (overtaking not allowed)

Figure 2 Bus trajectories for different extreme $\gamma$ cases for $k=0.25$ 


\subsection{Tests under various degrees of front-bus preference and $k$ levels}

In this section, the impact of the spectrum of different degrees of front-bus preference are tested. For this we utilise the total standard deviation of $\Delta_{m, n}$ for all services as illustrated in Figure 2 to illustrate the system regularity. Figure 3 is to show the max $\Delta_{m, n}$ of the system for the same range of parameter settings. In both figures we distinguish the case when overtaking is allowed (Figs. 3(a) and 4(a)) and when it is not allowed (Figs. 3(b) and 4(b)). Figure 5 then presents an explicit comparison between overtaking and no-overtaking cases. All the tests are carried out under four different arriving-to-loading levels $k=0.25,0.3,0.35$ and 0.4 .

\subsubsection{Overtaking is allowed}

As is shown in Figures 3(a) and 4(a), the standard deviation of $\Delta_{m, n}$ reaches its minimum at $\gamma=0$ in most cases, while the maximum is obtained for $\gamma=0.5$ in all cases. A similar tendency could be observed for max $\Delta_{m, n}$. Accordingly, equally-split queueing strategy $(\gamma=$ 0.5 ) is unfavourable from a system perspective, as it will disturb the service regularity most. This is understandable as $\gamma=0.5$ means that buses, once they are bunched, will always depart from stops at the same time as discussed before. In line with our observations from Figure 2, zero front-bus preference $(\gamma=0)$ maintains the service evenness well. We notice that under all $k$-levels $\gamma=1$ can perform almost as well as $\gamma=0$ if overtaking is allowed.

\subsubsection{Overtaking is not allowed}

As is shown in Figures 3(b) and 4(b), except for some points of max $\Delta_{m, n}$, the indices reach their minimum when all the passengers board the back bus, which confirms our previous observations. Besides, as is illustrated in Figure 3(b), unevenness of services are nonlinearly aggravated with the increase of $\gamma$ especially for high $k$ levels. For the max $\Delta_{m, n}$, we can observe some fluctuations in Figure 4(b) illustrating the complexity of the system.

\subsubsection{Comparison between overtaking and no-overtaking case}

The comparison between overtaking and no-overtaking is illustrated in Figure 5. We utilise the ratios between the indices of the no-overtaking case and the overtaking case to analyse the discrepancies between these two cases. Except for $k=0.4$, bus services perform the same before $\gamma$ exceeds 0.5 , because overtaking rarely occurs in line with Table 1 . The only case when overtaking can occur also with $\gamma<0.5$ is when three buses are bunched and if $k$ is high. Compared with the overtaking case, we observe a significant increase in the standard deviation 
of $\Delta_{m, n}$ for the no-overtaking case, which illustrates the importance of allowing for overtaking to maintain service regularity. When $\gamma>0.5$, the higher the front-bus preference is, the more improvement could be obtained by allowing for overtaking. Further, the higher $k$, the higher the contribution of overtaking to improve the service which is shown in both Figures 5(a) and 5(b). 


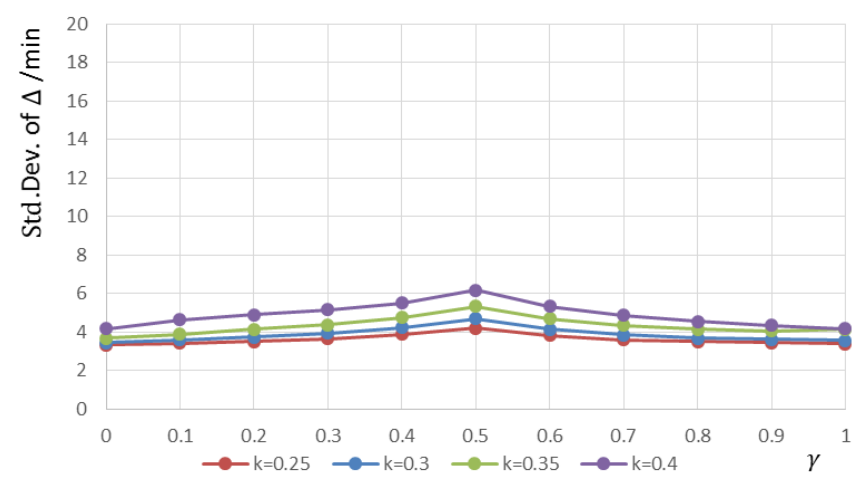

(a) with overtaking

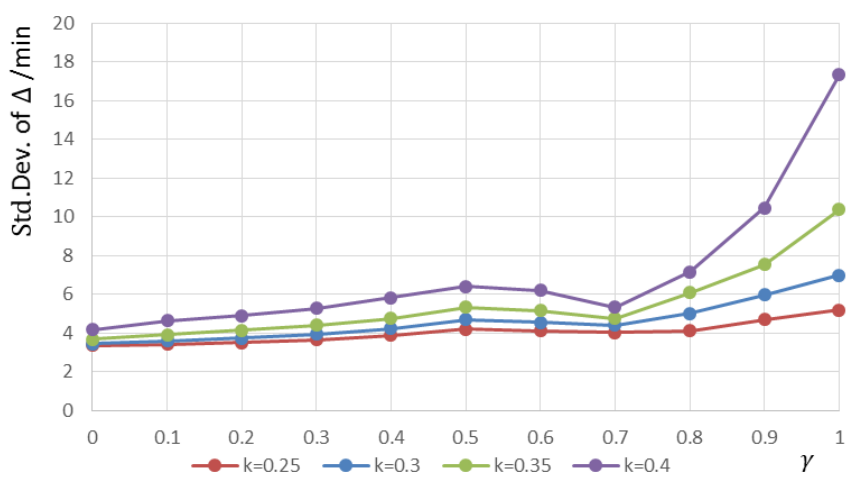

(b) without overtaking

Figure 3 Standard deviation of $\Delta_{m, n}$ for different $\gamma$ and $k$

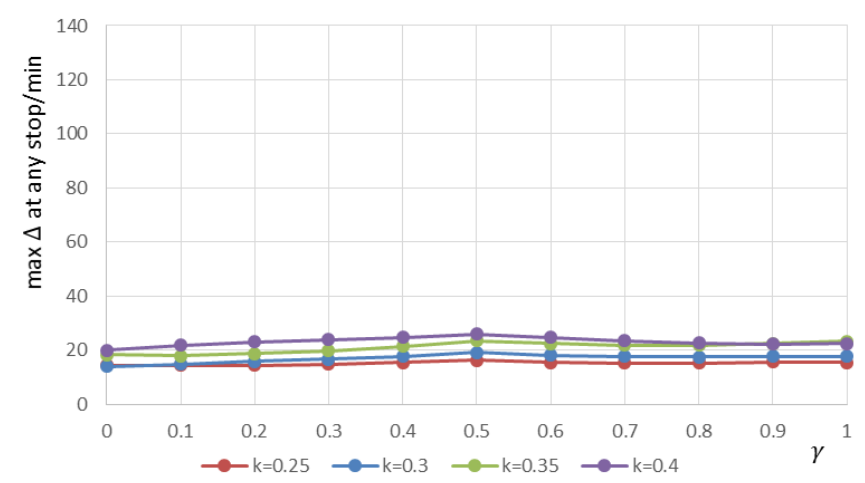

(a) with overtaking

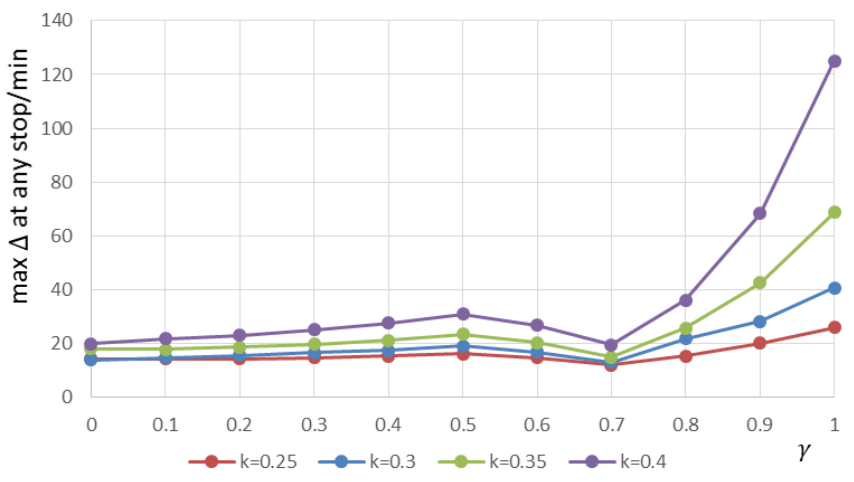

(b) without overtaking

Figure $4 \operatorname{Max} \Delta_{m, n}$ for different $\gamma$ and $k$

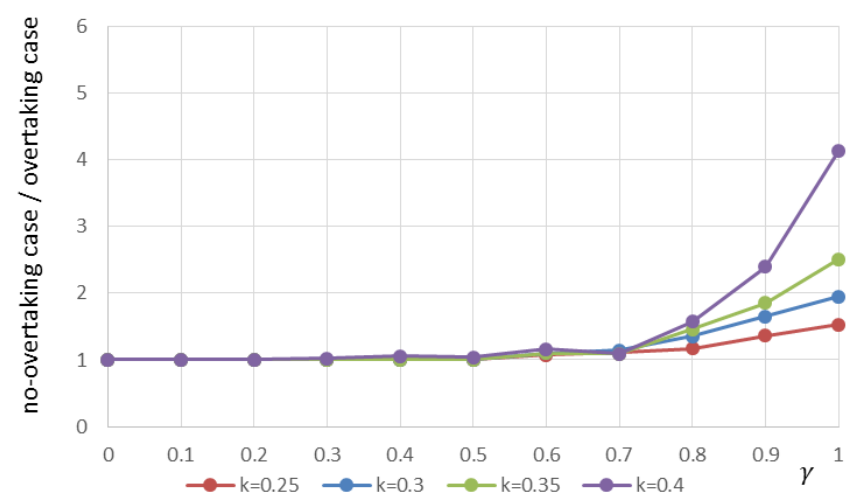

(a) Standard deviation of $\Delta_{m, n}$

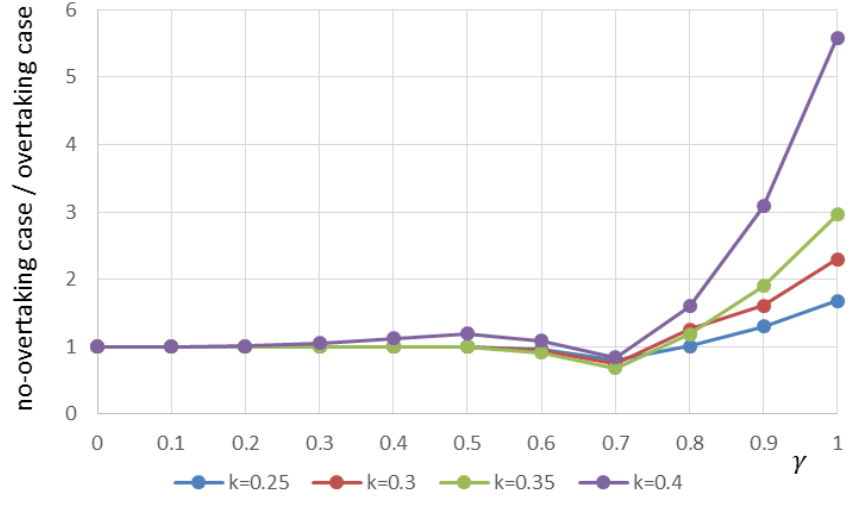

(b) $\operatorname{Max} \Delta_{m, n}$

Figure 5 Comparison between no-overtaking and overtaking cases for different $\gamma$ and $k$ 


\subsection{Test with different initial delay patterns}

In this section, the effect of initial delay on the service regularity is investigated. For this test, standard deviation of $\Delta_{m, n}$ is considered as the primary indicator, in particular the changes in service regularity along with the increase in initial delay. The $k$ ratio is held at 0.25 to indicate a realistic boarding demand and overtaking is permitted. For the front-bus preference, 3 critical points: $\gamma=0, \gamma=0.5$ and $\gamma=1$ are selected in this test.

As is illustrated in Figure 6, initial delay is generated for the 2nd bus at the 2nd stop and its size is varied from $0.2 \mathrm{~min}$ to $8 \mathrm{~min}$. Service regularity performs increasingly unfavorably until the initial delay exceeds $2 \mathrm{~min}$. Given an initial delay between 2 and $6 \mathrm{~min}$, service regularity appears to be independent of the size of initial delay, although there are some fluctuations, which indicates that the overtaking strategy can contribute to the control of service regularity. When the initial delay is too large and exceeds the headway a lot, the regularity tends to be out of control.

To illustrate the effect of multiple delays further tests are made where another exogenous delay is given to the $3 \mathrm{rd}$ bus also at the $2 \mathrm{nd}$ stop. The size of delay of the 2 nd bus is fixed at $2 \mathrm{~min}$ and that of the $3 \mathrm{rd}$ bus is varied from $0 \mathrm{~min}$ to $8 \mathrm{~min}$. As is shown in Figure 7, the indicator drops suddenly when the delay of $3 \mathrm{rd}$ bus is between $1 \mathrm{~min}$ and $2 \mathrm{~min}$, strongly indicating that the following bus should be held by a period that is close to the delay of the leading bus at the stop. Similarly, a drop in service regularity reduction can be observed when the delay of 3rd bus is between $7 \mathrm{~min}$ and $8 \mathrm{~min}$, though the reduction is reduced due to the overall larger delay.

Our tests with multiple delays further support the conclusion derived in Section 6.3. Adding $\gamma$ as additional dimension to the graph, Figure 8 shows that $\gamma=0.5$ remains the worst solution. Besides, the service regularity is increasingly weakened when $\gamma$ varies from 0 to 0.5 and continuously improves when it increases from 0.5 to 1 . 


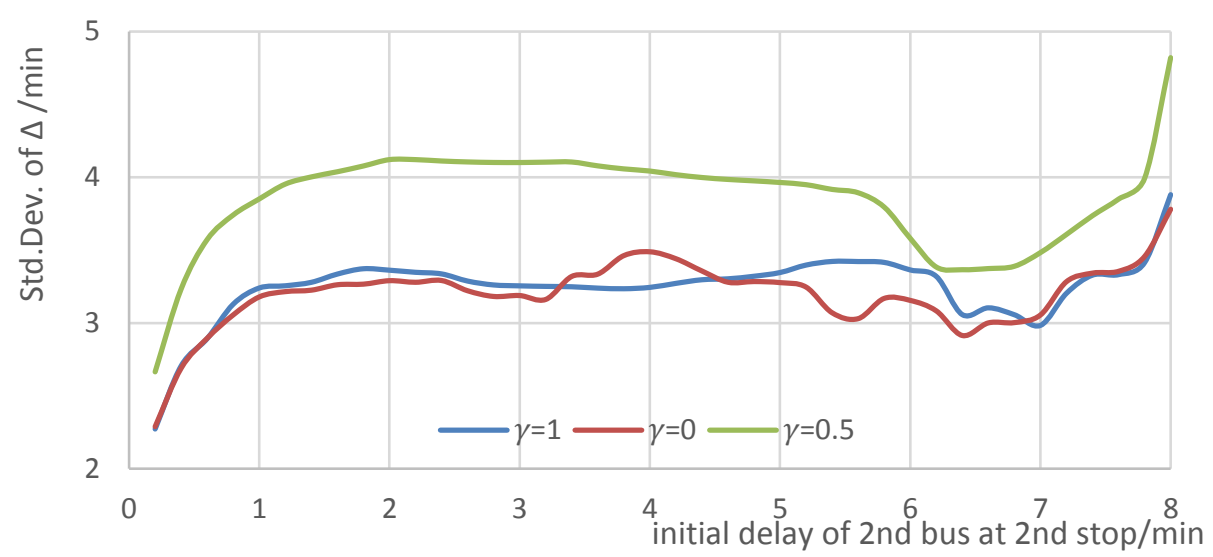

Figure 6 Service regularity with an initial delay of 2 nd bus at 2nd stop

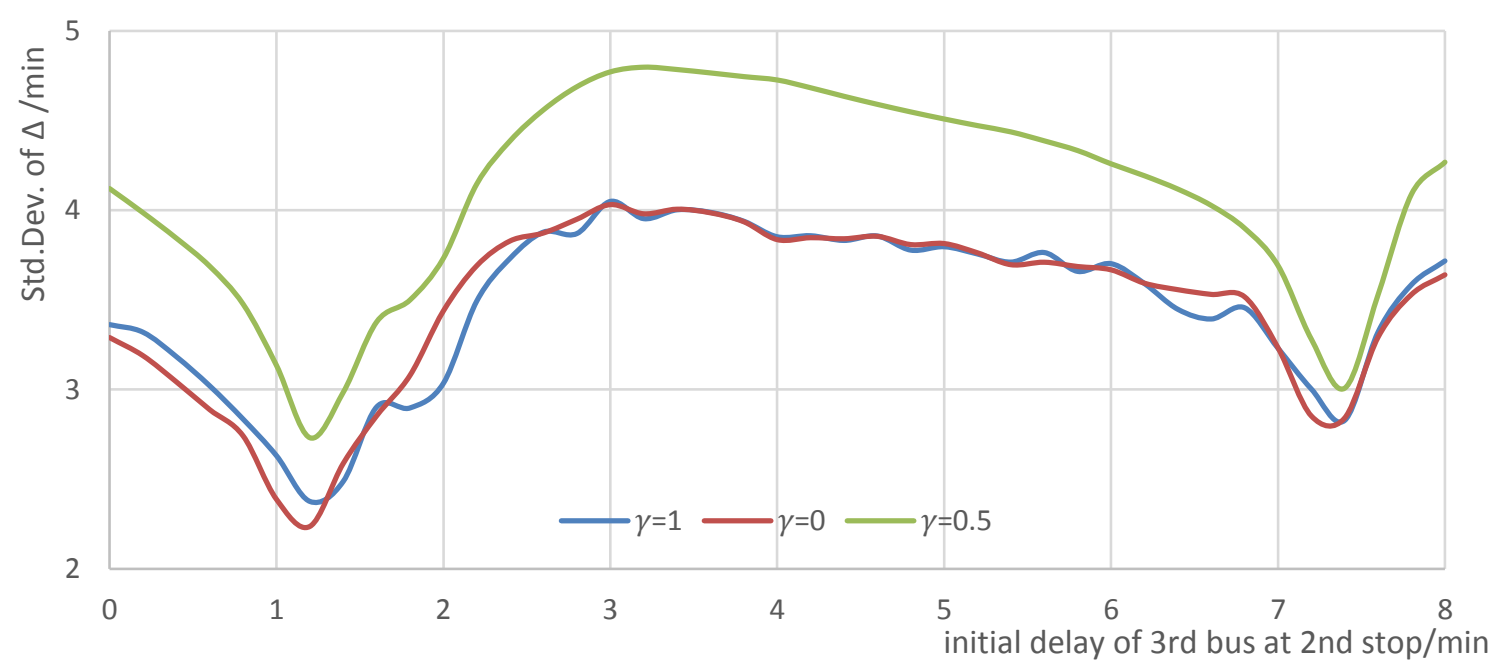

Figure 7 Service regularity with initial delays of 2 nd and 3 rd bus at 2 nd stop

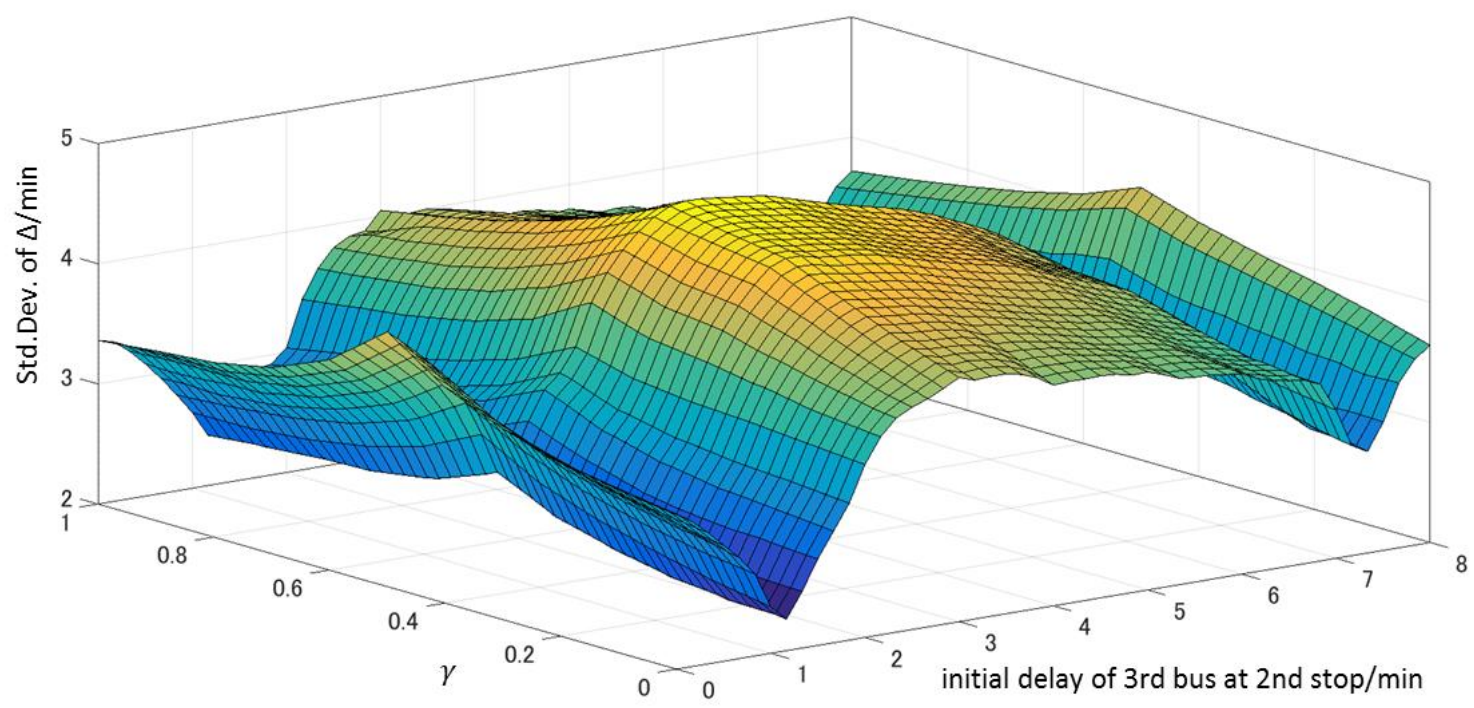

Figure 8 Service regularity under different initial delay patterns and various degrees of frontbus preference 


\section{Conclusions and Further Work}

This paper contributes to modelling and explaining the effect of passenger behavior at stops on bus bunching. A passenger behavior parameter to denote the preference to board the front bus has been introduced. We then discuss the different arrival and departure patterns that can occur at a bus stop and solve the resulting problems to obtain the bus dwell times. The dwell time formulations are then implemented into a recursive set of state equations to model the bunching effects along a line.

We evaluate the resulting service regularity given an initial disturbance to one or two buses at one of the first stops along a corridor. For this we obtain the standard deviation and maximum headways between two bus departures. Through our case study we derive some general conclusions:

We demonstrate that the operator's overtaking policy is of significance if passengers show no propensity to take the back bus of two buses boarding at the same time. That is, overtaking can be considered a counter-measure to bunching if front-bus preference is high or the arriving-toloading burden is heavy. When the front-bus preference exceeds 0.5 , the higher the front-bus preference is, the more improvement could be obtained by allowing for overtaking.

More generally, the case that passengers prefer to board the back bus tends to provide a better service. Such a back-bus preference can be encouraged or enforced by the bus drivers and operators. If the driver of the front bus stops boarding passengers as soon as another bus arrives, $\gamma=0$ will be enforced. From a fairness perspective though this might be frustrating for passengers that have been at the front of the queue and might now end up further in the back of the queue of passengers boarding the second bus. Therefore, as an operational policy it might be important to explain to the passengers through notifications at congested stops that taking the latter bus is for the "good of the system". In other words the problem described in this paper can also be interpreted as a conflict between user equilibrium and system optimal solution. The user equilibrium solution (of the myopic traveller not being able to consider effects further downstream) corresponds to $\gamma=0.5$ as travellers will in that case form equal queues for both buses so that all travellers leave the bus stop at the same time and no traveller can improve his departure time by swapping queue. As our graphs show $\gamma=0.5$ is though clearly not the optimal solution for the system which tends to be $\gamma=0$.

This work is meant to model and illustrate the effect of $\gamma$ but clearly a number of issues have 
not been considered that we believe should be addressed in further work. We note that the frontbus preference degree of the passengers might differ depending on the position of passengers in the queue and whether they arrive before bus arrival or while the bus is boarding passengers. Considering such behavior would require replacing our constant $\gamma$ with one that is a function of arrival time and queue length.

More important for practical applications is though that bus choice in bunched situations will depend on remaining available spaces in the buses. Capacity issues as well as alighting issues are neglected in this paper. In further work, we are considering adding these factors into our model which would result in the $\gamma$ parameter becoming a function of the available bus capacity as well as further revisions to describe the total dwell time considering boarding as well as alighting. Other further work directions are consideration of passenger behaviour and overtaking as part of existing service control strategies that have been described in our literature review.

\section{Acknowledgment}

The authors acknowledge various comments received by colleagues at Kyoto University as well as funding received from JSPS Grant "Kiban B” 26289174.

\section{References}

Bartholdi, J. J., and D. D. Eisenstein. A self-coordinating bus route to resist bus bunching. Transportation Research, Part B, Vol. 46, 2012, pp. 481-491.

Cats, O., A. Larijani, W. Burghout and H.N. Koutsopoulos. Impacts of holding control strategies on transit performance: A bus simulation model analysis. Transportation Research Record, No. 2584, 2012, pp. 51-58.

Chen, X., L. Yu, Y. Zhang and J. Guo. Analyzing urban bus service reliability at the stop, route, and network levels. Transportation Research, Part A, Vol. 43, 2009, pp. 722-734.

Daganzo, C. F. A headway-based approach to eliminate bus bunching: Systematic analysis and comparisons. Transportation Research, Part B, Vol. 43, Issue. 10, 2009, pp. 913-921.

Daganzo, C.F. and J. Pilachowski. Reducing bunching with bus-to-bus cooperation. Transportation Research, Part B, Vol. 45, 2011, pp. 267-277.

Eberlein, X.J., M.H.M. Wilson and D. Bernstein. The holding problem with real-time information available. Transportation Science, Vol. 35, No. 1, 2001, pp. 1-18.

Fernández, R. Modelling public transport stops by microscopic simulation. Transportation 
Research, Part C, Vol.18, Issue. 6, 2010, pp. 856-868.

Fonzone, A., J.-D. Schmöcker and R. Liu. A model of bus bunching under reliability-based passenger arrival patterns. Transportation Research, Part C, 59, 2015, pp. 164-182.

Gu, W., Y. Li, M. Cassidy and J. Griswold. On the capacity of isolated, curbside bus stops. Transportation Research, Part B, Vol. 45, 2011, pp. 714-723.

Hernández, D., J.C. Muñoz, R. Giesen and F. Delgado. Analysis of real-time control strategies in a corridor with multiple bus services. Transportation Research, Part B, Vol. 78, 2015, pp. 83-105.

Hickman, M.D. An analytic stochastic model for the transit vehicle holding problem. Transportation. Science, Vol. 35, No. 3, 2001, pp. 215-237.

Hollander, Y. and R. Liu. Estimation of the distribution of travel times by repeated simulation. Transportation Research Part C, Vol. 16, 2008, pp. 212-231.

Liu, R. and S. Sinha. S. Modelling urban bus service and passenger reliability. Selected proceedings of the $3^{\text {rd }}$ International Symposium on Transportation Network Reliability, The Hague, 2007.

Newell, G.F. Control of pairing of vehicles on a public transportation route, two vehicles, one control point. Transportation Science, Vol. 8, No. 3, 1974, pp. 248-264.

Newell, G.F. and R.B. Potts. Maintaining a bus schedule. Proceedings of $2^{\text {nd }}$ Australian Road Research Board, No. 2, 1964, pp. 388-393.

Osuna, E.E. and G.F. Newell. Control strategies for an idealized bus system. Trans. Sci. Vol. 6, No. 1, 1972, pp. 52-71.

Pilachowski, J.M. An Approach to Reducing Bus Bunching. PhD Thesis, University of California, Berkeley, 2009.

Schmöcker, J.-D., W. Sun, R. Liu and A. Fonzone. Bus Bunching Along a Corridor Served by Two Lines. Transportation Research, Part B, Vo. 93, 2016, pp. 300-316.

Sun, L., A. Tirachini, K.W. Axhausen, A. Erath and D.H. Lee. Models of bus boarding and alighting dynamics. Transportation Research, Part A, Vol. 69, 2014, pp. 447-460. 